\title{
High effectiveness of pandemic influenza A (H1N1) vaccination in healthcare workers from a Portuguese hospital
}

\author{
José Torres Costa $\cdot$ Rui Silva $\cdot$ Margarida Tavares $\cdot$ \\ Albert Nienhaus
}

Received: 26 March 2011 / Accepted: 11 October 2011 / Published online: 2 November 2011

(C) The Author(s) 2011. This article is published with open access at Springerlink.com

\begin{abstract}
Objectives Vaccination of healthcare workers (HCWs) was made a high priority during the phase six pandemic of the novel influenza A H1N1 (pH1N1) virus. We surveyed adherence to $\mathrm{pH} 1 \mathrm{~N} 1$ vaccination and the incidence of pH1N1 infection between vaccinated and unvaccinated HCWs.

Methods Employees at the S. João Hospital in Porto, Portugal, were offered $\mathrm{pH} 1 \mathrm{~N} 1$ vaccinations free of charge. Pandemrix ${ }^{\circledR}$ was the vaccine administered. As part of the pandemic plan, employees with influenza-like symptoms (ILS) were called upon to take an RT-PCR H1N1 test. If the test results were positive, they had to stay off work for at least 7 days. Sociodemographic data, vaccination status, contact with infectious patients, ILS and pH1N1 test results were documented in a standardised manner.

Results The survey population comprised 5,592 employees. The vaccination rate was $30.8 \%(n=1,720)$ for $\mathrm{pH} 1 \mathrm{~N} 1$
\end{abstract}

J. T. Costa R. Silva

Occupational Health Division, S. João Hospital,

EPE, Porto, Portugal

J. T. Costa $\cdot$ R. Silva

Allergy Division, S. João Hospital, EPE, Porto, Portugal

J. T. Costa $\cdot$ R. Silva $\cdot$ M. Tavares

Medical School, Oporto University, Porto, Portugal

M. Tavares

Infectious Diseases Division, S. João Hospital,

EPE, Porto, Portugal

A. Nienhaus $(\square)$

University Medical Centre Hamburg-Eppendorf, Institute for Health Service Research in Dermatology and Nursing, Martinistraße 52, 20246 Hamburg, Germany e-mail: a.nienhaus@uke.de and $50.4 \%(n=2,819)$ for the $2009 / 2010$ seasonal trivalent inactivated influenza vaccine (TIV). One mild anaphylactic reaction occurred after $\mathrm{pH} 1 \mathrm{~N} 1$ vaccination. Minor local side effects occurred more often after $\mathrm{pH} 1 \mathrm{~N} 1$ vaccination than after 2009/2010 seasonal TIV (38.0\% vs. $12.3 \%$ ). Pandemic H1N1 infection was diagnosed in 97 HCWs (1.7\%). Compared to employees with no regular patient contact, nurses $(2.8 \%)$ had the highest risk of $\mathrm{pH} 1 \mathrm{~N} 1$ infection (adjusted OR 3.8; 95\% CI 1.2-6.8). Vaccination reduced the $\mathrm{pH} 1 \mathrm{~N} 1$ infection risk (OR $0.12 ; 95 \%$ CI $0.05-0.29$ ). Vaccine effectiveness was 90.4\% (95\% CI 73.5-97.3\%).

Conclusion Vaccination reduced the $\mathrm{pH} 1 \mathrm{~N} 1$ infection risk considerably. The pandemic plan to contain the $\mathrm{pH} 1 \mathrm{~N} 1$ infection was successful. Nurses had the highest risk of pH1N1 infection and are therefore a target group for vaccination measures.

Keywords Pandemic influenza A H1N1 · Healthcare workers · Vaccination

$\begin{array}{ll}\text { Abbreviations } \\ \text { pH1N1 } & \text { Pandemic influenza A H1N1 } \\ \text { ILS } & \text { Influenza-like symptoms } \\ \text { TIV } & \text { Trivalent inactivated influenza vaccine } \\ \text { HCWs } & \text { Healthcare workers } \\ \text { OR } & \text { Odds ratio } \\ \text { RT-PCR } & \text { Real-time reverse transcriptase-polymerase } \\ & \text { chain reaction }\end{array}$

\section{Introduction}

In the middle of April 2009, cases of infection with a new influenza virus were detected in Mexico and southern California (MMWR 2009). This virus was later identified 
as an H1N1 influenza virus, with six genes derived from triple-reassortant North American swine virus lineages and two genes (encoding neuraminidase and matrix proteins) derived from Eurasian swine virus lineages (Garten et al. 2009). It rapidly spread to many countries around the world, prompting the World Health Organization (WHO) to declare a phase six global influenza pandemic on 11 June 2009 (WHO 2009a). At that time, 74 countries had reported over 27,000 cases of pandemic influenza A H1N1 (pH1N1) and 141 deaths (WHO 2009b). Three months later, the virus had spread to over 170 countries and was estimated to have caused 3,486 deaths (WHO 2009c).

The development of an effective vaccine against the new strain of the virus and the subsequent implementation of a large-scale immunisation campaign was considered one of the most effective ways to control the pandemic. The immunisation of healthcare workers (HCWs) was given high priority in order to protect the healthcare infrastructure (WHO 2009d).

In Portugal, a national vaccination plan against the pH1N1 virus was implemented, using the vaccine Pandemrix $^{\circledR}$, containing $3.75 \mu \mathrm{g}$ of haemagglutinin (General Directorate of Health 2009). It was available from the second half of October 2009. According to national guidelines, the vaccine was to be given to priority groups including HCWs and emergency medical services personnel. The aim of our study was to analyse the incidence of pH1N1 influenza and the effectiveness of $\mathrm{pH} 1 \mathrm{~N} 1$ vaccination in HCWs at a Portuguese tertiary referral teaching hospital.

\section{Methods}

The pH1N1 vaccination was offered to all HCWs working at S. João Hospital in Porto, Portugal, during the influenza season 2009/2010. Vaccination started on 26 October 2009. No predetermined end date for the vaccination campaign was given. On 10 January 2010, the last HCW was vaccinated. Participants were asked to remain under observation for $60 \mathrm{~min}$ after vaccination so that any side effects could be identified. The observation period was limited to $1 \mathrm{~h}$ because if severe side effects, i.e. anaphylactic reaction, occur they will be apparent within the first hour after vaccination. Furthermore, HCWs were asked to actively report any side effects of the vaccination to the vaccination desk in an informal manner. In addition, HCWs were either asked directly during their next visit to the OSHdepartment or contacted by phone within 3 months of their pH1N1 vaccination and asked whether any side effects occurred. For this interview, a semi-standardised survey was used containing a list of potential side effects such as soreness, redness or swelling at injection site, muscle aches, or fever.
Seasonal vaccination 2009/2010 commenced on 14 September 2009 using the trivalent inactivated influenza vaccine (TIV) CHIROFLU ${ }^{\circledR}$ from Novartis Lab. In those participants with a previous seasonal vaccination, side effects of the vaccination were assessed at the time of the pH1N1 vaccination. Both $\mathrm{pH} 1 \mathrm{~N} 1$ and seasonal vaccination were given free of charge to the HCWs and information regarding the vaccinations was disseminated in a similar fashion within the hospital.

According to the contingency plan for pH1N1 control, HCWs with influenza-like symptoms (ILS) were attended to by a specialised physician at the $\mathrm{pH} 1 \mathrm{~N} 1$ task force unit created in the Emergency Department. The task force examined HCWs with ILS and offered antiviral treatment. This treatment was only available in the hospital. A nasopharyngeal or oropharyngeal tissue swab was taken from each HCW with ILS for the detection of the $\mathrm{pH} 1 \mathrm{~N} 1$ virus, using the real-time reverse transcriptase-polymerase chain reaction (RT-PCR) method.

All HCWs were monitored by the Occupational Health Division and requested to stay at home until the test results were known. The HCWs were allowed to return to their usual workplace if the result of the RT-PCR was negative and the symptoms had improved. However, if the RT-PCR was positive, the HCWs had to stay at home for a period of at least 7 days. This sick leave did not result in any loss of income or benefits regardless of the RT-PCR result. The analysis is restricted to ILS or pH1N1 infections that occurred after $\mathrm{pH} 1 \mathrm{~N} 1$ vaccination was available. Before 26 October, only eleven cases of ILS and two cases of pH1N1 infection were registered. Before the swab was taken, symptoms were recorded and HCWs were asked whether they had had contact with patients or other persons with ILS.

The contingency plan for pH1N1 control not only recommended vaccination, antiviral treatment and social distancing but also emphasised disinfection, hand-washing and use of masks in order to prevent transmission. However, these latter aspects were not part of this analysis.

Data analysis was performed with SPSS, version 13. Adjusted odds ratio (OR) and 95\% confidence interval (CI) for putative risk factors for ILS or pH1N1 infection were calculated. Pearson's Chi-square test was employed for categorical data using $\alpha<0.05$ as the significance level. The number of prevented cases of pH1N1 influenza was calculated by subtracting the observed cases in vaccinated HCWs from the expected cases had the HCWs not been vaccinated. Vaccine effectiveness (VE) was estimated using the following formula: $\mathrm{VE}=(\mathrm{PPV}-\mathrm{PCV}) /(\mathrm{PPV}(1-\mathrm{PCV}))$ $\times 100 \%$, where PPV is the proportion vaccinated in the cohort and PCV the proportion of vaccinated cases (Farrington 1993). For this calculation, an HCW was considered vaccinated when the onset of symptoms started later than 1 week after the vaccination. The ethical integrity 
Fig. 1 Flow chart of the study population. Pearson's Chisquare test for $\mathrm{pH} 1 \mathrm{~N} 1$ infection yes or no depending on $\mathrm{pH} 1 \mathrm{~N} 1$ vaccination in the group with seasonal TIV $(p<0.0001)$ and without seasonal TIV $(p=0.004)$

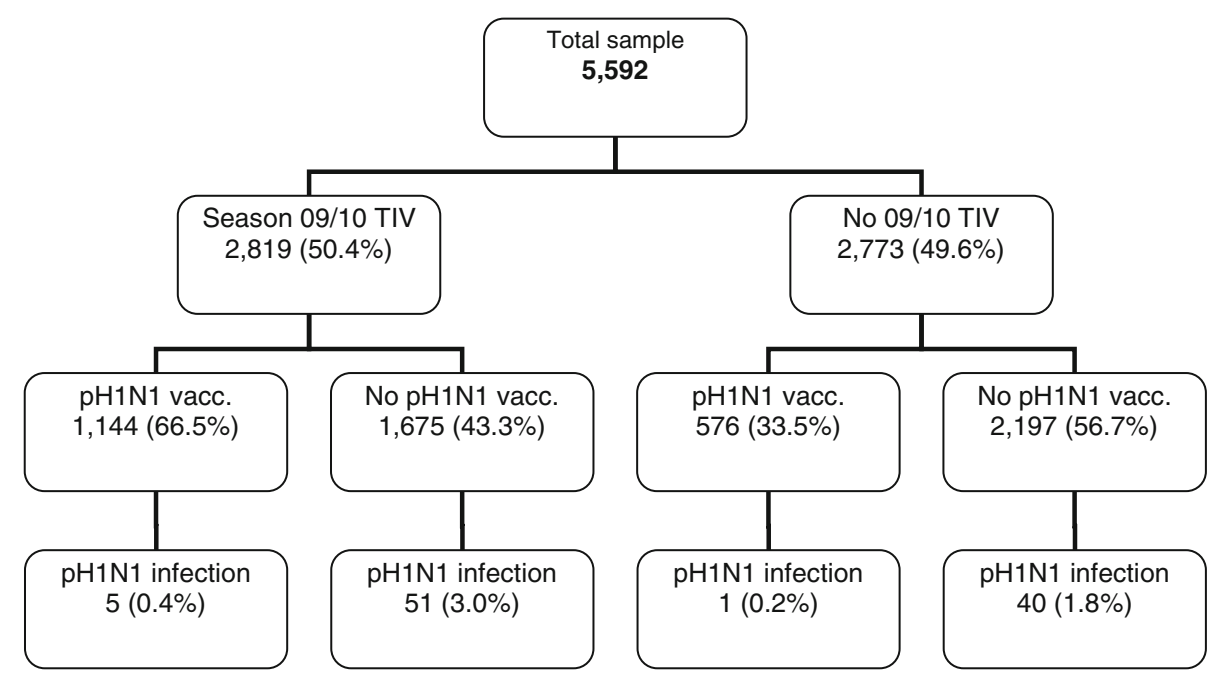

Table 1 Description of the study population $(n=5,592)$

\begin{tabular}{|c|c|c|}
\hline & $N$ & $\%$ \\
\hline Female & 4,042 & 72.3 \\
\hline \multicolumn{3}{|l|}{ Age } \\
\hline$\leq 30$ years & 1,471 & 26.3 \\
\hline $31-40$ years & 1,724 & 30.8 \\
\hline $41-50$ years & 1,236 & 22.1 \\
\hline$>50$ years & 1,161 & 20.8 \\
\hline Pregnancy & 52 & 0.9 \\
\hline \multicolumn{3}{|l|}{ Profession } \\
\hline Nurses & 1,982 & 35.4 \\
\hline Physicians & 1,393 & 24.9 \\
\hline Auxiliary staff & 1,273 & 22.8 \\
\hline Administration or others & 944 & 16.9 \\
\hline \multicolumn{3}{|l|}{ Vaccination } \\
\hline $\mathrm{pH} 1 \mathrm{~N} 1$ & 1,720 & 30.8 \\
\hline Seasonal 09/10 TIV & 2,819 & 50.4 \\
\hline Seasonal 08/09 TIV & 2,127 & 38.0 \\
\hline \multicolumn{3}{|l|}{ Seasonal influenza } \\
\hline No vaccination & 2,172 & 38.8 \\
\hline TIV in $2008 / 2009$ & 601 & 10.7 \\
\hline TIV in $2009 / 2010$ & 1,293 & 23.1 \\
\hline TIV in both seasons & 1,526 & 27.3 \\
\hline Influenza-like symptoms (ILS) & 245 & 4.4 \\
\hline Confirmed pH1N1 infection & 97 & 1.7 \\
\hline
\end{tabular}

of the study was confirmed by the pH1N1 task force (General Directorate of Health 2009) and HCWs gave their informed consent to an anonymous analysis of their data.

\section{Results}

The study sample comprises 5,592 HCWs with and without regular patient contact (Fig. 1). In total, 1,720 HCWs were
Table 2 Seasonal TIV and 2009 pH1N1 vaccination rates by profession

\begin{tabular}{lrrrrr}
\hline Profession & \multicolumn{2}{l}{ TIV } & & \multicolumn{2}{l}{ pH1N1-vacc. } \\
\cline { 2 - 3 } & $N$ & $\%$ & & $N$ & $\%$ \\
\hline Nurses & 1,238 & 62.5 & & 601 & 30.3 \\
Physicians & 650 & 46.7 & & 611 & 43.9 \\
Auxiliary staff & 602 & 47.3 & & 252 & 19.8 \\
Administration or others & 329 & 34.9 & & 256 & 27.1 \\
\hline
\end{tabular}

vaccinated against $\mathrm{pH} 1 \mathrm{~N} 1$ (30.8\%), including 52 pregnant HCWs (Table 1). $50.4 \%$ of the study population received seasonal TIV for the season 2009/2010. Nurses had the highest vaccination rate $(62.5 \%)$ for seasonal TIV but only the second highest rate (30.3\% compared to $43.9 \%$ in physicians) for $\mathrm{pH} 1 \mathrm{~N} 1$ vaccination (Table 2).

After pH1N1 vaccination, one woman experienced an anaphylactic reaction with dizziness and hypotension lasting a few minutes. No further complications were observed during the first hour after vaccination and no side effects warranting medical attention were reported. After $\mathrm{pH} 1 \mathrm{~N} 1$ vaccination, myalgia $(6.9 \%)$, mild local reaction $(38.0 \%)$ and strong local reaction (1.9\%) were reported to the vaccination desk (Table 3). No complications occurred in the 52 pregnant participants. Assessed retrospectively, 83.4\% reported no side effects from the seasonal TIV, $12.3 \%$ mild local reactions and $2.9 \%$ myalgia. Strong local reactions $(0.7 \%)$, fatigue $(0.3 \%)$, fever $(0.3 \%)$, headaches $(0.1 \%)$ and lymph node swelling $(0.1 \%)$ were seldom. Therefore, more side effects were reported after $\mathrm{pH} 1 \mathrm{~N} 1$ vaccination than after the 2009/2010 seasonal TIV.

Between 26 October 2009 and 2 March 2010, 245 HCWs with ILS (4.4\%) were referred to the pH1N1 task force in the Emergency Department (Table 1). Of these, pH1N1 virus infection was confirmed in 97 cases (39.6\%). 
Table 3 Side effects of seasonal vaccination $09 / 10$ in those 1,144 HCWs who obtained $\mathrm{pH} 1 \mathrm{~N} 1$ vaccination and side effects of $\mathrm{pH} 1 \mathrm{~N} 1$ vaccination

\begin{tabular}{llrllr}
\hline Side effects* & \multicolumn{2}{l}{$2009 / 2010$ seasonal TIV } & & \multicolumn{2}{l}{$\mathrm{pH} 1 \mathrm{~N} 1$} \\
\cline { 2 - 3 } \cline { 6 - 7 } & $N$ & $\%$ & & $N$ & $\%$ \\
\hline No side effects & 954 & 83.4 & & 994 & 57.8 \\
Fatigue & 3 & 0.3 & & 10 & 0.6 \\
Headache & 1 & 0.1 & & 3 & 0.2 \\
Temperature $>38^{\circ} \mathrm{C}$ & 3 & 0.3 & & 9 & 0.5 \\
Myalgia & 33 & 2.9 & & 119 & 6.9 \\
Lymph node swelling & 1 & 0.1 & & - & \\
Mild local reaction & 141 & 12.3 & & 654 & 38.0 \\
Strong local reaction & 8 & 0.7 & & 32 & 1.9 \\
Total & 1,144 & 100.0 & & 1,720 & 100.0 \\
\hline
\end{tabular}

* Multiple responses were possible

Indeterminate results were observed in two out of 245 examinations $(0.8 \%)$. After performing a second test, one case remained indeterminate.

The peak in ILS and pH1N1 infection in HCWs came in the 49th week of 2009. ILS occurred less often in pH1N1vaccinated HCWs (OR 0.7; 95\% CI 0.51-0.95), while the seasonal TIV showed no protective effect against ILS (OR 1.0; 95\% CI 0.79-1.36). Gender was not associated with ILS (Table 4). Younger workers were more likely to present with ILS (OR for $\leq 30$ years: 2.7; 95\% CI 1.69-4.42). After adjusting for vaccination, nurses (OR 2.5; 95\% CI 1.53-4.09) and physicians (OR 2.0; 95\% CI 1.21-3.41) had a higher risk of developing ILS than administrators.

Out of the 97 pH1N1 infections, 91 (94\%) occurred in non-vaccinated HCWs and two $(2 \%)$ in HCWs vaccinated less than a week before the onset of symptoms. Overall, pH1N1 incidence was $1.7 \%$ of all HCWs, affecting $0.3 \%$ of those vaccinated and $2.4 \%$ of those not vaccinated (Table 5). The seasonal TIV did not protect against $\mathrm{pH} 1 \mathrm{~N} 1$ infection (OR 1.5; 95\% CI 0.98-2.27) and neither did consecutive seasonal TIV in 2008 and 2009 (Table 1) (data not shown). Young HCWs were more often affected (OR for $\leq 30$ years: 6.6; 95\% CI 2.57-16.8, Table 5). Nurses had an increased risk of pH1N1 infection (OR 2.7; 95\% CI 1.116.37), while physicians had an increased but not statistically significant risk (OR 1.8; 95\% CI 0.71-4.62). A total of $41 \mathrm{pH} 1 \mathrm{~N} 1$ infections would have been expected in the vaccinated HCWs if they had not been vaccinated. The number of prevented cases is therefore 35 (41 expected minus six observed cases). The number to vaccinate in order to prevent one case of pH1N1 influenza was 49 in this particular cohort. Vaccine effectiveness (VE) was $90.4 \%$ (95\% CI 73.5-97.3\%).

Sixty-two $(64 \%)$ of the pH1N1 infected HCWs had had known contact with a pH1N1 infected individual and
Table 4 Logistic regression for putative risk factors of influenza-like symptoms (ILS)

\begin{tabular}{|c|c|c|c|c|}
\hline \multirow[t]{3}{*}{ Variable } & \multicolumn{2}{|l|}{ ILS } & \multirow[t]{3}{*}{ OR } & \multirow[t]{3}{*}{$95 \% \mathrm{CI}$} \\
\hline & Neg. & Pos. & & \\
\hline & $N(\%)$ & $N(\%)$ & & \\
\hline \multicolumn{5}{|c|}{ pH1N1 vaccination } \\
\hline No & $3,690(95.3)$ & $182(4.7)$ & 1 & - \\
\hline Yes & $1,657(96.3)$ & $63(3.7)$ & 0.7 & $0.51-0.95$ \\
\hline \multicolumn{5}{|c|}{ Seasonal TIV 09/10 } \\
\hline No & $2,658(95.9)$ & $115(4.1)$ & 1 & - \\
\hline Yes & 2,689 (95.4) & $130(4.6)$ & 1.0 & $0.79-1.36$ \\
\hline \multicolumn{5}{|l|}{ Gender } \\
\hline Female & $3,856(95.4)$ & $186(4.6)$ & 1 & - \\
\hline Male & $1,491(96.2)$ & $59(3.8)$ & 0.9 & $0.64-1.18$ \\
\hline \multicolumn{5}{|l|}{ Age (years) } \\
\hline$\leq 30$ & $1,379(93.7)$ & $92(6.3)$ & 2.7 & $1.69-4.42$ \\
\hline $31-40$ & $1,638(95.0)$ & $86(5.0)$ & 2.3 & $1.42-3.72$ \\
\hline $41-50$ & $1,191(96.4)$ & $45(3.6)$ & 1.8 & $1.01-3.03$ \\
\hline$>50$ & $1,139(98.1)$ & $22(1.9)$ & 1 & - \\
\hline \multicolumn{5}{|l|}{ Profession } \\
\hline Nurses & $1,854(93.5)$ & $128(6.5)$ & 2.5 & $1.53-4.09$ \\
\hline Physicians & $1,330(95.5)$ & $63(4.5)$ & 2.0 & $1.21-3.41$ \\
\hline Auxiliary staff & $1,239(97.3)$ & $34(2.7)$ & 1.1 & $0.63-1.95$ \\
\hline $\begin{array}{l}\text { Administration } \\
\text { or others }\end{array}$ & 924 (97.9) & $20(2.1)$ & 1 & - \\
\hline
\end{tabular}

another 17 HCWs (17.5\%) had had contact with symptomatic individuals. Fifty out of 79 potential sources of infection $(63 \%)$ were patients in the hospital. The most frequent symptoms associated with $\mathrm{pH} 1 \mathrm{~N} 1$ infection were muscle or joint pain (85\%), coughing (78\%), fever (77\%), headache $(61 \%)$ and sore throat $(40 \%)$. The disease was benign in its evolution in all cases.

\section{Discussion}

To our knowledge, this is the first study to analyse the incidence of pH1N1 infection and vaccine effectiveness in HCWs in the 2009/2010 season. According to our data, nurses were the most affected group. Most of the known infectious contacts were with patients. The vaccination rate was 30.8 , and $94 \%$ of the pH1N1 infections were observed in the unvaccinated HCWs. Vaccination reduced the attack rate of $\mathrm{pH} 1 \mathrm{~N} 1$ from 2.4 to $0.3 \%$. Vaccination may have prevented $35 \mathrm{pH} 1 \mathrm{~N} 1$ infections in this particular cohort and pandemic season. Calculated vaccine effectiveness was $90.4 \%$ and therefore high.

The pandemic plan at S. João Hospital ensured that no HCWs who took sick leave due to ILS suffered any loss of 
Table 5 Logistic regression for putative risk factors for $\mathrm{pH} 1 \mathrm{~N} 1 \mathrm{infec}-$ tion

\begin{tabular}{|c|c|c|c|c|}
\hline \multirow[t]{3}{*}{ Variables } & \multicolumn{2}{|l|}{ pH1N1 } & \multirow[t]{3}{*}{ OR } & \multirow[t]{3}{*}{$95 \%$ CI } \\
\hline & Neg. & Pos. & & \\
\hline & $N(\%)$ & $N(\%)$ & & \\
\hline \multicolumn{5}{|c|}{ pH1N1 vaccination } \\
\hline No & 3,781 (97.6) & $91(2.4)$ & 1 & - \\
\hline Yes & 1,714 (99.7) & $6(0.3)$ & 0.12 & $0.05-0.29$ \\
\hline \multicolumn{5}{|c|}{ Seasonal TIV 09/10 } \\
\hline No & $2,732(98.5)$ & $41(1.5)$ & 1 & - \\
\hline Yes & $2,763(98.0)$ & $56(2.0)$ & 1.5 & $0.98-2.27$ \\
\hline \multicolumn{5}{|l|}{ Gender } \\
\hline Female & $3,972(98.3)$ & $70(1.7)$ & 1 & - \\
\hline Male & $1,523(98.3)$ & $27(1.7)$ & 1.1 & $0.72-1.82$ \\
\hline \multicolumn{5}{|l|}{ Age (years) } \\
\hline$\leq 30$ & $1,421(96.6)$ & $50(3.4)$ & 6.6 & $2.57-16.8$ \\
\hline $31-40$ & $1,692(98.1)$ & $32(1.9)$ & 3.8 & $1.47-9.95$ \\
\hline $41-50$ & $1,226(99.2)$ & $10(0.8)$ & 1.7 & $0.59-5.09$ \\
\hline$>50$ & 1,156 (99.6) & $5(0.4)$ & 1 & - \\
\hline \multicolumn{5}{|l|}{ Profession } \\
\hline Nurses & 1,926 (97.2) & $56(2.8)$ & 2.7 & $1.11-6.37$ \\
\hline Physicians & $1,374(98.6)$ & $19(1.4)$ & 1.8 & $0.71-4.62$ \\
\hline Auxiliary staff & 1,257 (98.7) & $16(1.3)$ & 1.4 & $0.55-3.65$ \\
\hline $\begin{array}{l}\text { Administration } \\
\text { or others }\end{array}$ & 938 (99.4) & $6(0.6)$ & 1 & - \\
\hline
\end{tabular}

income or benefits. This was granted to all HCWs with ILS regardless of whether it was caused by $\mathrm{pH} 1 \mathrm{~N} 1$ infection or not. Furthermore, antiviral treatment was only offered to those who reported to the Emergency Department. These two circumstances increased the likelihood of reporting ILS. Therefore, this could well have neutralised any potential reluctance to report ILS to the pandemic task force. However, asymptomatic infections could not be detected by testing HCWs with ILS only and infections with mild symptoms are likely to have been underreported. This limitation renders it likely that the incidence of pH1N1 infection was underestimated in our cohort. However, underreporting was most likely non-differential and therefore did not influence the estimate of vaccine effectiveness. In a previous German study, vaccine effectiveness was estimated to be $96.8 \%$ in the general population (Wichmann et al. 2010). Therefore, the vaccine effectiveness we found in HCWs (90.4\%) was slightly lower. For the United Kingdom, a lower vaccine effectiveness of $71 \%$ was reported (Hardelid et al. 2011). Vaccine effectiveness increased to $72 \%$ if vaccination was assumed to be effective 2 weeks after injection instead of one, as we assumed. In a European multicentre study, vaccine effectiveness was $78.4 \%$ in persons < 65 years (Valenciano et al. 2011). Therefore, our observation is well within the range of vaccine effectiveness found in other populations.

The vaccination rate against the $\mathrm{pH} 1 \mathrm{~N} 1$ virus $(30.8 \%)$ was significantly lower than for the seasonal TIV in the same year $(50.4 \%)$. Similar ratios were also described by other authors, with pH1N1 vaccination levels varying, depending on country and institution, between 15 and 37\% (Wicker et al. 2010; Sullivan et al. 2010). The main cause of pandemic vaccine refusal was concern about its safety and the belief that it was not needed (Rachiotis et al. 2010; SteelFisher et al. 2010; Ofri 2009). Our data suggest that the $\mathrm{pH} 1 \mathrm{~N} 1$ vaccination was safe and effective. Side effects were more frequent after $\mathrm{pH} 1 \mathrm{~N} 1$ vaccination than after seasonal TIV. However, they were mostly minor local reactions. As a limitation of the study, it should be noted that underreporting of side effects of seasonal TIV is possible if side effects of seasonal TIV discouraged HCWs from accepting pH1N1 vaccination. Underreporting of side effects caused by the pH1N1 vaccination is not likely because in addition to active reporting of side effects to the vaccination desk a survey on side effects was performed with all HCWs who received the vaccination. The frequency of side effects we observed was similar to that described in an Italian HCWs study, which reported pain at the injection site (43.4\%) as the most frequent adverse reaction (Amodio et al. 2011). As increased knowledge and awareness could well have an improved impact on adherence to vaccination schemata, our data might help to convince $\mathrm{HCWs}$ to take part in vaccination campaigns for the coming influenza seasons (Hofmann et al. 2006).

Seasonal influenza vaccination was not effective against pH1N1 infection. This corroborates the findings of Jefferies et al. (2011) from New Zealand. The authors conclude that 2009 seasonal influenza vaccination had no protective effect against $\mathrm{pH} 1 \mathrm{~N} 1$ infection amongst HCWs.

The major limitation of our study is that only participants with ILS were tested for pH1N1 infection. There might have been underreporting of ILS, and a certain number of pH1N1 infections might have been asymptomatic and therefore remained unnoticed. However, surveys on the incidence of $\mathrm{pH} 1 \mathrm{~N} 1$ infections describe infection rates very similar to our findings in HCWs who were not vaccinated (Reed et al. 2009, 2011; Santos et al. 2010; Brammer et al. 2011). This corroborates the infection rates we found and renders serious underreporting unlikely. Furthermore, the sensitivity and specificity of the RT-PCR for $\mathrm{pH} 1 \mathrm{~N} 1$ are suitable for diagnostic use but they are not perfect (Ellis et al. 2009). Tests for antibodies after the infection or ILS were not performed in order to confirm the pH1N1 infection. This might have resulted in false positive or false negative results. However, this should have led to non-differential misclassification and dilution of the preventive effect of $\mathrm{pH} 1 \mathrm{~N} 1$ vaccination. Therefore, the vaccine 
effectiveness observed in our study is unlikely to be overestimated. Side effects of the pH1N1 vaccination were directly observed during the first hour after vaccination. It should be noted that information on other side effects was based on informal reports to the vaccination desk and a semi-standardised survey either in person or over the phone. Therefore, underestimation of the incidence of side effects after $\mathrm{pH} 1 \mathrm{~N} 1$ vaccination is possible but not likely to introduce a significant bias.

\section{Conclusions}

Vaccine effectiveness seemed to be high in HCWs during the influenza A H1N1 season 2009/2010. The pandemic plan to contain pandemic influenza A H1N1, with its various methods, was successful. The use of vaccines significantly reduced the expected number of illnesses. Nurses had the highest risk of $\mathrm{pH} 1 \mathrm{~N} 1$ infection and are therefore a target group for vaccination measures.

Acknowledgments We would like to thank the HCWs who participated in this study. No funds were received for this study.

Conflict of interest The authors declare that they have no conflict of interest.

Open Access This article is distributed under the terms of the Creative Commons Attribution Noncommercial License which permits any noncommercial use, distribution, and reproduction in any medium, provided the original author(s) and source are credited.

\section{References}

Amodio E, Anastasi G, Marsala MG, Torregrossa MV, Romano N, Firenze A (2011) Vaccination against the 2009 pandemic influenza A (H1N1) among healthcare workers in the major teaching hospital of Sicily (Italy). Vaccine 29(7):1408-1412

Brammer L, Blanton L, Epperson S et al (2011) Surveillance for influenza during the 2009 influenza A (H1N1) pandemic-United States, April 2009-March 2010. Clin Infect Dis 52(Suppl 1):S27S35

Ellis J, Iturriza M, Allen R et al (2009) Evaluation of four real-time PCR assays for detection of influenza A (H1N1) virus. Euro Surveill 14(22)

Farrington CP (1993) Estimation of vaccine effectiveness using the screening method. Int J Epidemiol 22(4):742-746

Garten RJ, Davis CT, Russell CA et al (2009) Antigenic and genetic characteristics of swine-origin 2009 A (H1N1) influenza viruses circulating in humans. Science 325:197-201

General Directorate of Health (2009) Vaccination campaign against the pandemic influenza (H1N1) 2009 infection. Information bulletin no. 17, 14 October 2009 (Direcção-Geral da Saúde (2009)
Campanha de vacinação contra a infecção pelo vírus da gripe pandémica (H1N1). Circular Normativa no 17, 14 October 2009)

Hardelid P, Fleming DM, McMenamin J et al (2011) Effectiveness of pandemic and seasonal influenza vaccine in preventing pandemic influenza A (H1N1) 2009 infection in England and Scotland 2009-2010. Euro Surveill 16(2):pii19763

Hofmann F, Ferracin C, Marsh G, Dumas R (2006) Influenza vaccination of healthcare workers: a literature review of attitudes and beliefs. Infection 34:142-147

Jefferies S, Earl D, Berry N et al (2011) Effectiveness of the 2009 seasonal influenza vaccine against pandemic influenza A (H1N1) 2009 in healthcare workers in New Zealand. Euro Surveill 16(2)

MMWR (2009) Swine influenza A (H1N1) infection in two childrenSouthern California, March-April 2009. MMWR Morb Mortal Wkly Rep 58:400-402

Ofri D (2009) The emotional epidemiology of H1N1 influenza vaccination. N Engl J Med 361:2594-2595

Rachiotis G, Mouchtouri VA, Kremastinou J, Gourgoulianis K, Hadjichristodoulou C (2010) Low acceptance of vaccination against the 2009 pandemic influenza A (H1N1) among healthcare workers in Greece. Euro Surveill 15(6):pii=19486

Reed C, Angulo FJ, Swerdlow DL et al (2009) Estimates of the prevalence of pandemic (H1N1) 2009, Unites States April-July. Emerg Infect Dis 15(12):2004-2007

Reed C, Angulo FJ, Biggerstaff M, Swerdlow DL, Finelli L (2011) Influenza-like illness in the community during the emergence of 2009 pandemic influenza a (H1N1)-survey of 10 states, April 2009. Clin Infect Dis 52(Suppl 1):S90-S93

Santos CD, Bristow RB, Vorenkamp JV (2010) Which health care workers were most affected during the spring 2009 H1N1 pandemic? Disaster Med Public Health Prep 4(1):47-54

SteelFisher GK, Blendon RJ, Bekheit M, Lubell K (2010) The public's response to the $2009 \mathrm{H} 1 \mathrm{~N} 1$ influenza pandemic. N Engl J Med $362(22): \mathrm{e} 65$

Sullivan J, Jacobson M, Dowdle R, Poland GA (2010) 2009 H1N1 influenza. Mayo Clin Proc 85:64-76

Valenciano M, Kissling E, Cohen JM et al (2011) Estimates of pandemic influenza vaccine effectiveness in Europe, 2009-2010: results of influenza monitoring vaccine effectiveness in Europe (I-MOVE) multicentre case-control study. PLoS Med 8(1):e1000388

Wichmann O, Stocker P, Poggensee G, Altmann D, Walter D, Hellenbrand W, Krause G, Eckmanns T (2010) Pandemic influenza A(H1N1) 2009 breakthrough infections and estimates of vaccine effectiveness in Germany 2009-2010. Euro Surveill 15(18)

Wicker S, Rabenau HF, Bias H, Groneberg DA, Gottschalk R (2010) Influenza A (H1N1) 2009: impact on Frankfurt in due consideration of healthcare and public health. J Occup Med Toxicol 5:10

World Health Organization (WHO) (2009a) New influenza A/H1N1 virus: global epidemiological situation, June 2009. Wkly Epidemiol Rec 84:249-257

World Health Organization (WHO) (2009b) Information for the media-influenza A (H1N1): WHO announces pandemic alert phase 6 , of moderate severity. WHO press, release:06

World Health Organization (WHO) (2009c) Pandemic (H1N1) 2009update 66. WHO 09:18

World Health Organization (WHO) (2009d) WHO recommendations on pandemic (H1N1) 2009 vaccines. Pandemic (H1N1) 2009 briefing note 2 . WHO 07:13 